\title{
SWEEDLER'S TWO-COCYCLES AND GENERALIZATIONS OF THEOREMS ON AMITSUR COHOMOLOGY
}

\author{
BY
}

\author{
DAVE RIFFELMACHER
}

\begin{abstract}
For any (not necessarily commutative) algebra $C$ over a commutative ring $k$ Sweedler defined a cohomology set, denoted here by $\mathcal{I}^{2}(C / k)$, which generalizes Amitsur's second cohomology group $H^{2}(C / k)$. In this paper, if $I$ is a nilpotent ideal of $C$ and $\bar{C} \equiv C / I$ is $k$-projective, a natural bijection $\mathcal{T}^{2}(C / k) \stackrel{\sim}{\rightarrow} \mathcal{T}^{2}(\bar{C} / k)$ is established. Also, when $k \subset B$ are fields and $C$ is a commutative $B$-algebra, the sequence $\{1\} \rightarrow$ $\mathcal{C}^{2}(B / k) \stackrel{\iota^{*}}{\rightarrow} \mathcal{C}^{2}(C / k) \stackrel{r}{\rightarrow} \mathcal{S C}^{2}(C / B)$ is shown to be exact if the natural map $C \otimes_{k} C \rightarrow C \otimes_{B} C$ induces a surjection on units, $b^{*}$ is induced by the inclusion, and $r$ is the "restriction" map.
\end{abstract}

Introduction. Given a commutative algebra $C$ over a commutative ring $k$, Amitsur introduced a cochain complex and demonstrated that in certain cases the second cohomology group $H^{2}(C / k)$ of this complex is isomorphic to the Brauer group of similarity classes of central separable $k$-algebras split by $C$ [1]. Sweedler generalized the notion of an Amitsur two-cocycle to noncommutative algebras $C$ and has shown that various sets of certain equivalence classes of (generalized) two-cocycles classify various types of $k$-algebras [8]. In this paper we study several functorial properties of the full set, denoted here by $\mathcal{H}^{2}(C / k)$, of certain equivalence classes of Sweedler's two-cocycles.

Sweedler's basic definitions are recalled in $\$ 1$. $\$ 2$ provides a generalization of a theorem of Rosenberg and Zelinsky [6, Proposition 3.3] which states that under suitable faithfully flatness conditions the Amitsur cohomology of a commutative algebra is not changed by factoring out a nilpotent ideal. We prove that, if $I$ is a nilpotent ideal of the (not necessarily commutative) $k$-algebra $C$ and $\bar{C} \equiv C / I$ is $k$-projective, the natural projection $C \rightarrow \bar{C}$ induces a bijection $\mathcal{H}^{2}(C / k) \stackrel{\sim}{\rightarrow} \mathcal{H}^{2}(\bar{C} / k)$. In $\$ 3$ we restrict our attention to commutative algebras. If $k \subseteq B$ are fields, $C$ is a commutative $B$-algebra, and the natural map $C \otimes_{k} C \rightarrow C \otimes_{B} C$ induces a surjection on units, we prove there is an exact sequence

$$
\{1\} \rightarrow \mathcal{H}^{2}(B / k) \stackrel{\iota^{*}}{\rightarrow} \mathcal{H}^{2}(C / k) \stackrel{r}{\rightarrow} \mathcal{H}^{2}(C / B)
$$

where $\iota$ * is induced by the inclusion $\iota: B \rightarrow C$ and $r$ is the "restriction" map.

Received by the editors February 8, 1977.

AMS (MOS) subject classifications (1970). Primary $18 \mathrm{H} 15$.

(C) 1979 American Mathematical Society $0002-9947 / 79 / 0000-0312 / \$ 03.75$ 
This sequence is a partial generalization and synthesis of two sequences Yuan used in his study of $p$-algebras [10, Theorems 3.4, 3.5].

1. Definitions. Let $C$ be an algebra over a commutative ring $k$ and let unadorned $\otimes$ represent $\otimes_{k}$. Following Sweedler [8] we call an element $\delta=\Sigma_{i} x_{i} \otimes y_{i}$ in $C \otimes C$ a $C$-one-cocycle if $\Sigma_{i} x_{i} \otimes 1 \otimes y_{i}=\Sigma_{i, j} x_{i} \otimes y_{i} x_{j} \otimes$ $y_{j}$. Two $C$-one-cocycles $\delta=\Sigma_{i} x_{i} \otimes y_{i}$ and $\eta=\Sigma_{i} u_{i} \otimes v_{i}$ are said to be equivalent if there is a unit $z$ in $C$ with $\Sigma_{i} z^{-1} x_{i} \otimes y_{i} z=\Sigma_{i} u_{i} \otimes v_{i}$. An element $\Sigma_{i} x_{i} \otimes y_{i}$ in $C \otimes C$ is called vertible if $\Sigma_{i} x_{i} \otimes y_{i}^{0}$ is invertible in $C \otimes C^{0}$. (Here $C^{0}$ is the opposite $k$-algebra.) We denote the set of equivalence classes of vertible $C$-one-cocycles by $\mathcal{F C}^{1}(C / k)$ or by $\mathcal{H}^{1}(C)$ if the ground ring is clear from the context.

A $C$-two-cocycle is an element $\sigma=\Sigma_{i} a_{i} \otimes b_{i} \otimes c_{i}$ in $C \otimes C \otimes C$ such that

$$
\sum_{i, j} a_{i} a_{j} \otimes b_{j} \otimes c_{j} b_{i} \otimes c_{i}=\sum_{i, j} a_{i} \otimes b_{i} a_{j} \otimes b_{j} \otimes c_{j} c_{i}
$$

and there is an $e_{\sigma}$ in $C$ with $\Sigma_{i} a_{i} e_{\sigma} b_{i} \otimes c_{i}=1 \otimes 1=\Sigma_{i} a_{i} \otimes b_{i} e_{\sigma} c_{i}$. We shall refer to these equations as the associativity and unitary conditions, respectively, since they arose to guarantee a certain construction $C^{\sigma}$ of Sweedler's would be a $k$-algebra. The $C$-two-cocycle $\sigma=\Sigma_{i} a_{i} \otimes b_{i} \otimes c_{i}$ is cohomologous to the $C$-two-cocycle $\tau=\Sigma_{i} r_{i} \otimes s_{i} \otimes t_{i}$ via $\delta=\Sigma_{i} x_{i} \otimes y_{i}$ in $C \otimes C$ if

$$
\begin{gathered}
\sum_{i} x_{i} e_{a} y_{i}=e_{\tau}, \\
\sum_{i, j} x_{i} a_{j} \otimes b_{j} \otimes c_{j} y_{i}=\sum_{i, j, l} r_{i} x_{j} \otimes y_{j} s_{i} x_{l} \otimes y_{l} t_{i} .
\end{gathered}
$$

If $\sigma$ is cohomologous to $\tau$ via a vertible $\delta$, we indicate this by $\sigma \sim \delta$ and say that $\sigma$ and $\tau$ are equivalent. We denote the set of equivalence classes of $C$-two-cocycles by $\mathcal{H}^{2}(C / k)$ or by $\mathcal{H}^{2}(C)$ if no confusion seems likely. Given a $C$-two-cocycle $\sigma=\Sigma_{i} a_{i} \otimes b_{i} \otimes c_{i}$ and a vertible element $\delta=\Sigma_{i} x_{i} \otimes y_{i}$ with verse $\bar{\delta}=\Sigma_{i} \bar{x}_{i} \otimes \bar{y}_{i}$ (i.e. $\sum_{i} \bar{x}_{i} \otimes \bar{y}_{i}^{0}$ is the inverse of $\Sigma_{i} x_{i} \otimes y_{i}^{g}$ ) we will of ten wish to consider the $C$-two-cocycle $\sigma_{1}=\sum_{i, j, l, m} \bar{x}_{i} a_{j} x_{l} \otimes y_{l} b_{j} x_{m} \otimes y_{m} c_{j} \bar{y}_{i}$ obtained by "altering" $\sigma$ by $\delta$. We shall say in this situation that $\sigma \sim \delta \sigma_{1}$ defines the $C$-two-cocycle $\sigma_{1}$. For example, if $C$ is commutative and $\sigma=\Sigma_{i} a_{i}$ $\otimes b_{i} \otimes c_{i}$ is a $C$-two-cocycle, the unitary condition implies that $e_{\sigma}$ is invertible (with inverse $\sum_{i} a_{i} b_{i} c_{i}$ ) and $\sigma \sim^{1 \otimes e_{o}^{-1}} \sigma_{1}$ defines a $C$-two-cocycle $\sigma_{1}$ with $e_{\sigma_{1}}=1$.

Any $k$-algebra map $C \stackrel{f}{\rightarrow} D$ induces a map

$$
C \otimes \stackrel{n}{*} \otimes C \stackrel{f^{n}}{\rightarrow} D \otimes \cdots \cdot \stackrel{n}{*} \otimes D
$$

given by $x_{1} \otimes \cdots \otimes x_{n} \rightarrow f\left(x_{1}\right) \otimes \cdots \otimes f\left(x_{n}\right)$ for each positive integer $n$ which we will denote by $f$ if no confusion seems likely. For $i=1,2$, we have 
an induced map on cohomology $\mathcal{H}^{i}(C) \stackrel{f^{*}}{\rightarrow} \mathcal{H C}^{i}(D)$.

2. A theorem of Rosenberg and Zelinsky. Throughout this section, $\mathcal{H}^{2}(D / k)$ will be denoted by $\mathcal{H}^{2}(D)$ for any $k$-algebra $D$. We prove:

THEOREM 2.1. Let $C$ be an algebra over a commutative ring $k$. Suppose $I$ is a nilpotent ideal of $C$ with $C / I$ projective as a $k$-module, and let $p: C \rightarrow C / I$ be the natural projection. Then $\mathcal{H}^{2}(C) \stackrel{p^{*}}{\rightarrow} \mathcal{H}^{2}(C / I)$ is bijective.

We begin by noting that Theorem 2.1 is equivalent to:

THEOREM 2.1'. Let $C$ be an algebra over a commutative ring $k$. Suppose $I$ is an ideal of $C$ with $I^{2}=\{0\}$ and $C / I$ is projective as a $k$-module. Let $p$ : $C \rightarrow C / I$ be the natural projection. Then $\mathcal{H}^{2}(C) \stackrel{p^{*}}{\rightarrow} \mathcal{H}^{2}(C / I)$ is bijective.

Proof THAT 2.1' IMPLIES 2.1. For any positive integer $n$ we have a commutative diagram

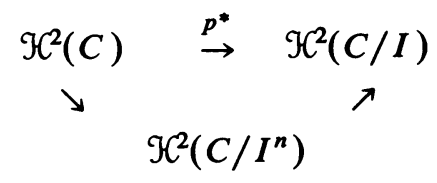

with each map induced by the appropriate canonical projection. Hence if $2.1^{\prime}$ is known, the general result 2.1 may be shown by an induction on the index of nilpotency of $I$.

Therefore we need only prove Theorem $2.1^{\prime}$ and accordingly in the remainder of this section we will assume $I^{2}=\{0\}$. With this assumption $I$ is a $C / I$-bimodule and we may view $C$ as a Hochschild extension of $C / I$ by $I$ [4]. Thus we have $C=C / I \oplus_{\psi} I$ as $k$-algebras where $\psi: C / I \otimes C / I \rightarrow I$ is a Hochschild two-cocycle. Recall that this means $C=C / I \oplus I$ as $k$-modules and the multiplication $*_{\psi}$ in $C / I \oplus_{\psi} I$ is given by $(a, x) *_{\psi}(b, y)=(a b, a y$ $+x b+\psi(a \otimes b))$ for $a, b$ in $C / I, x, y$ in $I$. The next theorem considers the special case of Theorem $2.1^{\prime}$ where $\psi$ is a trivial Hochschild two-cocycle.

TheOREM 2.2. Let $k, C, I$ and $p$ be as in Theorem 2.1'. Also assume $C=C / I \oplus_{\psi} I$ with $\psi$ a trivial Hochschild two-cocycle. Then $\mathcal{H}^{2}(C) \stackrel{p^{*}}{\rightarrow} \mathcal{H}^{2}(C / I)$ is bijective.

Proof. Since $\psi$ is trivial there is an algebra map $\iota: C / I \rightarrow C$ with $p \iota$ the identity of $C / I$. Denote the composite $\varphi$ by $q$. Then $q: C \rightarrow C$ is an algebra endomorphism satisfying $q^{2}=q$ and $\operatorname{ker}(q)=I$. It thus follows from Theorem 4.7 of [5] that any $C$-two-cocycle $\sigma$ is equivalent to its projection $q(\sigma)$ modulo $I$, which establishes the theorem.

We are now ready to prove Theorem $2.1^{\prime}$. The idea of the proof is to mimic the argument used to prove Theorem 4.7 of [5] while making allowance for the fact that $C / I$ is not, in general, a subalgebra of $C / I \oplus_{\psi} I$. 
$p^{*}$ is injective. Let $\sigma, \tau$ be $C$-two-cocycles with $p(\sigma)$ and $p(\tau)$ equivalent $C / I$-two-cocycles, i.e. there is an element $\delta$ in $C \otimes C$ with $p(\sigma)$ equivalent to $p(\tau)$ via $p(\delta)$. Since $I$ is nilpotent, $p(\delta)$ vertible implies that $\delta$ is vertible. Thus $\sigma \sim \delta \sigma_{1}$ defines a $C$-two-cocycle $\sigma_{1}$ equivalent to $\sigma$ with $p\left(\sigma_{1}\right)=p(\tau)$.

Hence we may assume

$$
\sigma=\tau+\sum_{i} f_{i} \otimes g_{i} \otimes h_{i}
$$

with $\sum_{i} f_{i} \otimes g_{i} \otimes h_{i}$ in $G=I \otimes C \otimes C+C \otimes I \otimes C+C \otimes C \otimes I$; that is, $\sigma \equiv \tau$ (modulo $\mathscr{G}$ ). We argue by induction on $n$ that if $\sigma \equiv \tau$ (modulo $\mathscr{G}^{n}$ ), there exist $C$-two-cocycles $\sigma_{1}, \tau_{1}$ with $\sigma_{1}$ equivalent to $\sigma, \tau_{1}$ equivalent to $\tau$, and $\sigma_{1} \equiv \tau_{1}$ (modulo $g^{n+1}$ ). This will be sufficient to establish the injectivity of $p^{*}$ because $g^{4}=\{0\}$.

Let $\tau=\Sigma_{i} a_{i} \otimes b_{i} \otimes c_{i}$ in (2.3) and define

$$
\delta=\sum_{i} p\left(a_{i} e_{\sigma}\right) b_{i} \otimes c_{i}+\sum_{i} p\left(f_{i} e_{\sigma}\right) g_{i} \otimes h_{i}
$$

Since

$$
\{p \otimes 1\}\left(\sum_{i} p\left(a_{i} e_{o}\right) b_{i} \otimes c_{i}\right)=1 \otimes 1
$$

and $I$ is nilpotent, $\Sigma_{i} p\left(a_{i} e_{\sigma}\right) b_{i} \otimes c_{i}$ is vertible. Denote its verse by $\Sigma_{i} x_{i} \otimes y_{i}$. $\sigma_{1} \sim^{\delta} \sigma$ and $\tau_{1} \sim^{\Sigma_{i} p\left(a_{i} e_{0}\right) b_{i} \otimes c_{i}} \tau$ define $C$-two-cocycles $\sigma_{1}, \tau_{1}$ with

$$
\begin{aligned}
\sigma_{1} \equiv & \tau_{1}-\sum_{i, j} a_{j} p\left(f_{i} e_{\sigma}\right) g_{i} \otimes h_{i} b_{j} \otimes c_{j}+\sum_{i} f_{i} \otimes g_{i} \otimes h_{i} \\
& +\sum_{i, j} p\left(f_{i} e_{\sigma}\right) g_{i} a_{j} \otimes b_{j} \otimes c_{j} h_{i}-\sum_{i, j} a_{j} \otimes b_{j} p\left(f_{i} e_{\sigma}\right) g_{i} \otimes h_{i} c_{j}
\end{aligned}
$$

(modulo $\left.g^{n+1}\right)$

where we have used

$$
\sum_{i} p\left(a_{i} e_{\sigma}\right) b_{i} \otimes c_{i} \equiv 1 \otimes 1 \equiv \sum_{i} x_{i} \otimes y_{i} \quad(\operatorname{modulo} C \otimes I+I \otimes C) .
$$

It follows from the associativity relation for $\sigma$ that

$$
\begin{aligned}
& \sum_{i, j} p\left(a_{i} f_{j} e_{\sigma}\right) g_{j} \otimes h_{j} b_{i} \otimes c_{i}+\sum_{i, j} p\left(f_{i} a_{j} e_{\sigma}\right) b_{j} \otimes c_{j} g_{i} \otimes h_{i} \\
& \equiv \sum_{i, j} p\left(a_{i} e_{\sigma}\right) b_{i} f_{j} \otimes g_{j} \otimes h_{j} c_{i}+\sum_{i, j} p\left(f_{i} e_{\sigma}\right) g_{i} a_{j} \otimes b_{j} \otimes c_{j} h_{i} \\
& \equiv \sum_{j} f_{j} \otimes g_{j} \otimes h_{j}+\sum_{i, j} p\left(f_{i} e_{\sigma}\right) g_{i} a_{j} \otimes b_{j} \otimes c_{j} h_{i} \quad\left(\text { modulo } g^{n+1}\right)
\end{aligned}
$$


so we may rewrite (2.4) as

$$
\begin{aligned}
& \sigma_{1} \equiv \tau_{1} \\
&+\sum_{i, j} p\left(f_{i} a_{j} e_{\sigma}\right) b_{j} \otimes c_{j} g_{i} \otimes h_{i} \\
&-\sum_{i, j} a_{j} \otimes b_{j} p\left(f_{i} e_{\sigma}\right) g_{i} \otimes h_{i} c_{j} \quad\left(\text { modulo } g^{n+1}\right)
\end{aligned}
$$

since $\Sigma_{i} f_{i} \otimes g_{i} \otimes h_{i}$ is in $G$ and $p(I)=\{0\}$. That is, we have shown

$$
\sigma_{1} \equiv \tau_{1}+\sum_{i} f_{i}^{\prime} \otimes g_{i}^{\prime} \otimes h_{i}^{\prime} \quad\left(\text { modulo } g^{n+1}\right)
$$

with $\Sigma_{i} f_{i}^{\prime} \otimes g_{i}^{\prime} \otimes h_{i}^{\prime}$ in $C \otimes I \otimes C+C \otimes C \otimes I$.

Suppose $\tau_{1}=\Sigma_{i} a_{i}^{\prime} \otimes b_{i}^{\prime} \otimes c_{i}^{\prime}$. If we now repeat the above procedure but "push down on the right", that is alter by

$$
\sum_{i} a_{i}^{\prime} \otimes b_{i}^{\prime} p\left(e_{\sigma_{1}} c_{i}^{\prime}\right)+\sum_{i} f_{i}^{\prime} \otimes g_{i}^{\prime} p\left(e_{\sigma_{1}} h_{i}^{\prime}\right)
$$

we obtain $C$-two-cocycles $\sigma_{2}, \tau_{2}$ with $\sigma_{2}$ equivalent to $\sigma_{1}, \tau_{2}$ equivalent to $\tau_{1}$, and

$$
\sigma_{2} \equiv \tau_{2}+\sum_{i} u_{i} \otimes v_{i} \otimes w_{i} \quad\left(\operatorname{modulo} g^{n+1}\right)
$$

with $v_{i}$ in $I$ for all $i$.

If $\tau_{2}=\Sigma_{i} r_{i} \otimes s_{i} \otimes t_{i}$, the associativity relation for $\sigma_{2}$ yields

$$
\begin{aligned}
& \sum_{i, j} r_{i} u_{j} \otimes v_{j} \otimes w_{j} s_{i} \otimes t_{i}+\sum_{i, j} u_{i} r_{j} \otimes s_{j} \otimes t_{j} v_{i} \otimes w_{i} \\
&\left.\equiv \sum_{i, j} r_{i} \otimes s_{i} u_{j} \otimes v_{j} \otimes w_{j} t_{i}+\sum_{i, j} u_{i} \otimes v_{i} r_{j} \otimes s_{j} \otimes t_{j} w_{i} \quad \text { (modulo } g^{n+1}\right) .
\end{aligned}
$$

Thus it follows that, modulo $\mathrm{g}^{n+1}$,

$$
\sum_{i, j} u_{i} r_{j} \otimes p\left(s_{j}\right) e_{\sigma_{2}} t_{j} v_{i} \otimes w_{i} \equiv \sum_{i, j} r_{i} \otimes p\left(s_{i} u_{j}\right) e_{\sigma_{2}} v_{j} \otimes w_{j} t_{i} \equiv 0
$$

Hence (2.5) implies $\sigma_{2} \equiv \tau_{2}$ (modulo $g^{n+1}$ ) which was to be shown.

$p^{*}$ is surjective. Let $J$ be the injective hull of $I$ as a $C / I$-bimodule. Define $\psi_{1}: C / I \otimes C / I \rightarrow J$ by $\psi_{1}(a \otimes b)=\psi(a \otimes b)$. Then $\psi_{1}$ is a trivial Hochschild two-cocycle since $J$ is injective $[3, \S I X .6]$ and, if we set $J^{2}=\{0\}$, $C / I \oplus_{\psi_{1}} J$ is a $k$-algebra with multiplication $(a, x) *_{\psi_{1}}(b, y)=(a b, a y+x b$ $\left.+\psi_{i}(a \otimes b)\right)$. We have a commutative diagram

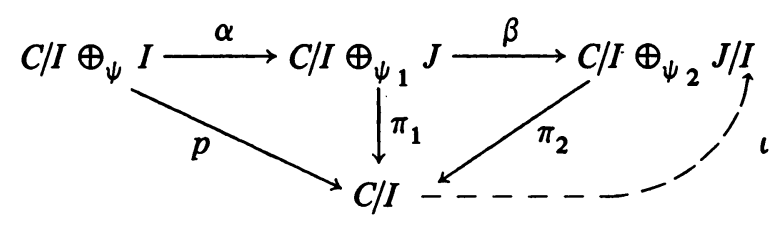


with $p, \pi_{1}, \pi_{2}$ the natural projections and $\alpha(a, x)=(a, x), \beta(a, y)=(a, y+$ $I), \iota(a)=(a, 0)$ for $a$ in $C / I, x$ in $I, y$ in $J$.

Let $\sigma$ be a $C / I \oplus_{\psi_{1}} J$-two-cocycle. Then $\beta(\sigma)$ is equivalent to $\iota \pi_{2} \beta(\sigma)=$ $\iota \pi_{1}(\sigma)$ via $\delta$ in

$$
1 \otimes 1+J / I \otimes\left\{C / I \oplus_{\psi_{2}} J / I\right\}+\left\{C / I \oplus_{\psi_{2}} J / I\right\} \otimes J / I
$$

by the proof of Theorem 2.2 .

Lift $\delta$ to $\delta^{\prime}$ in

$$
1 \otimes 1+J \otimes\left\{C / I \oplus_{\psi_{1}} J\right\}+\left\{C / I \oplus_{\psi_{1}} J\right\} \otimes J .
$$

$\delta^{\prime}$ is vertible since $I$ is nilpotent. Let $\sigma \sim^{\delta^{\prime}} \sigma^{\prime}$ define the $C / I \oplus_{\psi_{1}} J$-twococycle $\sigma^{\prime}$. Then

$$
\iota \pi_{2} \beta(\sigma) \sim^{\bar{\delta}} \beta(\sigma) \sim^{\beta\left(\delta^{\prime}\right)} \beta\left(\sigma^{\prime}\right),
$$

where $\bar{\delta}$ is the verse of $\delta$. Thus

$$
\iota \pi_{2} \beta(\sigma) \sim^{1 \otimes 1} \beta\left(\sigma^{\prime}\right),
$$

which implies $\sigma^{\prime}$ is in $\alpha\left(C / I \oplus_{\psi} I\right)$ and $\alpha^{*}$ is surjective.

We now have the commutative diagram

$$
\begin{array}{ccc}
\mathcal{H}^{2}\left(C / I \otimes_{\psi} I\right) & \stackrel{\alpha^{*}}{\rightarrow} & \mathcal{H}^{2}\left(C / I \otimes_{\psi_{1}} J\right) \\
p^{*} \searrow & \swarrow \pi_{1}^{*} \\
\mathcal{H C}^{2}(C / I) &
\end{array}
$$

Since $\psi_{1}$ is a trivial Hochschild two-cocycle, $\pi_{1}^{*}$ is bijective by Theorem 2.2 and hence it follows that $p^{*}$ is surjective. This completes the proof of Theorem 2.1' , and hence also of Theorem 2.1.

REMARKs. (1) If we restrict our attention to commutative algebras and invertible (i.e., Amitsur) $C$-two-cocycles, Theorem 2.1 is included in a theorem of Rosenberg and Zelinsky [6, Proposition 3.3].

(2) One may show that the induced map $\mathcal{H}^{1}(C) \stackrel{p^{*}}{\rightarrow} \mathcal{H}^{1}(C / I)$ is bijective by a similar (and far less tedious) argument.

3. An exact sequence. The object of this section is to establish that if $k \subseteq B$ are fields and $C$ is a commutative $B$-algebra there is an exact sequence of cohomology sets

$$
\{1\} \rightarrow \mathcal{H}^{2}(B) \stackrel{\iota^{*}}{\rightarrow} \mathcal{H}^{2}(C) \stackrel{r}{\rightarrow} \mathcal{H}^{2}(C / B)
$$

if the natural map $C \otimes C \rightarrow C \otimes_{B} C$ induces a surjection on the groups of units. Here $\iota: B \rightarrow C$ is the inclusion and $r$ is the "restriction" map arising from the natural projection $C \otimes C \otimes C \rightarrow C \otimes_{B} C \otimes_{B} C$. We begin with an easy extension of a theorem of Berkson [2, Theorem 1]. 
THEOREM 3.1. Let $k$ be a commutative ring and $C$ be a $k$-algebra which is free as a $k$-module. Then $\mathcal{H}^{1}(C)=\{1\}$.

Proof. Since $C$ is free as a $k$-module, we may write any vertible $C$-onecocycle as $\sum_{i=1}^{s} v_{i} \otimes w_{i}$ with $\left\{v_{i}\right\},\left\{w_{i}\right\}$ sets of linearly independent elements of $C$. Then the one-cocycle relation

$$
\sum_{i} v_{i} \otimes 1 \otimes w_{i}=\sum_{i, j} v_{i} \otimes w_{i} v_{j} \otimes w_{j}
$$

implies

$$
\sum_{i, j} v_{i} \otimes\left(w_{i} v_{j}-\delta_{i j}\right) \otimes w_{j}=0
$$

where $\delta_{i j}$ is the Kronecker delta. Since $\left\{v_{i}\right\}$ is linearly independent and $\left\{w_{i}\right\}$ is linearly independent we have $w_{i} v_{j}=\delta_{i j}$. Suppose $s>2$. Then

$$
\left(w_{1} \otimes v_{1}^{0}\right)\left(\sum_{i} v_{i} \otimes w_{i}^{0}\right)=1 \otimes 1^{0}=\left(w_{2} \otimes v_{2}^{0}\right)\left(\sum_{i} v_{i} \otimes w_{i}^{0}\right) .
$$

Hence, since inverses are unique and we are assuming that $\Sigma_{i} v_{i} \otimes w_{i}^{0}$ is invertible, $w_{1} \otimes v_{1}=w_{2} \otimes v_{2}$, a contradiction to the linear independence hypothesis. Thus $\Sigma_{i} v_{i} \otimes w_{i}=v_{1} \otimes w_{1}$. Since $v_{1} \otimes w_{1}$ is a $C$-one-cocycle, $v_{1} w_{1}=1$. Above we showed that $w_{1} v_{1}=1$. Hence $v_{1} \otimes w_{1}=v_{1} \otimes v_{1}^{-1}$, which is clearly equivalent to $1 \otimes 1$.

REMARK. The proof of Theorem 3.1 is Berkson's proof slightly modified to deal with the noncommutativity of $C$.

A close look at the proof of Theorem 3.1 yields a fact we will later need.

LEMMA 3.2. If $C$ is a commutative $k$-algebra and a free $k$-module, every C-one-cocycle is invertible.

Proof. The vertible hypothesis in Theorem 3.1 is needed since $w_{i} v_{j}=\delta_{i j}$.

Notation. If $k \subseteq L \subseteq C$ are commutative rings and $C$ is an $L$-algebra, we define $m_{L}: C \otimes C \rightarrow C \otimes_{L} C$ by

$$
m_{L}\left(\sum_{i} x_{i} \otimes y_{i}\right)=\sum_{i} x_{i} \otimes_{L} y_{i}
$$

and

$$
m_{L, L}=\left\{1 \otimes m_{L}\right\} \circ\left\{m_{L} \otimes 1\right\}: C \otimes C \otimes C \rightarrow C \otimes_{L} C \otimes_{L} C
$$

THEOREM 3.3. Let $k \subseteq L$ be fields and $C$ be a commutative L-algebra. If $\sigma, \tau$ are $L$-two-cocycles and $\sigma \sim^{\delta} \tau$ with $\delta$ a vertible element of $C \otimes C$, then $\delta=\left(\alpha \otimes \alpha^{-1}\right) \delta^{\prime}$ with $\delta^{\prime}$ in $L \otimes L$ and $\alpha$ anit of $C$.

Proof. Since $L$ is commutative we may without loss of generality (cf. $\S 1$ ) assume $e_{\sigma}=1=e_{\tau} \cdot m_{L, L}$ applied to (1.1) shows that $m_{L}(\delta)$ is a one-cocycle 
because $\sigma, \tau$ are in $L \otimes L \otimes L$. By Theorem 3.1 and Lemma $3.2 m_{L}(\delta)=\alpha$ $\otimes_{L} \alpha^{-1}$ for some $\alpha$ in $C$ and hence $\delta=\alpha \otimes \alpha^{-1}+\Sigma_{i} d_{i} \otimes e_{i}$ with $\left\{d_{i}\right\}$ $k$-linearly independent, $\left\{e_{i}\right\} k$-linearly independent, and $\Sigma_{i} d_{i} \otimes_{L} e_{i}=0$. Starting again with (1.1), applying the map $1 \otimes m_{L}$ and substituting $\alpha \otimes \alpha^{-1}$ $+\Sigma_{i} d_{i} \otimes e_{i}$ for $\delta$, we find that

$$
\sum_{i} d_{i} \otimes 1 \otimes_{L} e_{i}=\sum_{i} d_{i} \otimes e_{i} \alpha \otimes_{L} \alpha^{-1}
$$

A straightforward application of linear algebra yields $e_{i}=m_{i} \alpha^{-1}$ for all $i$, with $m_{i}$ in $L$. Similarly $d_{i}=n_{i} \alpha$ for all $i$, with $n_{i}$ in $L$. Therefore

$$
\delta=\left(\alpha \otimes \alpha^{-1}\right)\left(1 \otimes 1+\sum_{i} n_{i} \otimes m_{i}\right)
$$

which was to be shown.

An immediate consequence of Theorem 3.3 is

Corollary. Let $k \subseteq L$ be fields and $C$ be a commutative L-algebra. Then the inclusion $\iota: L \rightarrow C$ induces an injection $\mathcal{H}^{2}(L) \stackrel{\iota^{*}}{\rightarrow} \mathcal{H}^{2}(C)$.

The next theorem is the key result we need to prove exactness at $\mathcal{H}^{2}(C)$ in the purportedly exact sequence (ES) exhibited at the beginning of this section. First, however, we need a preliminary lemma.

LEMMA 3.4. Let $k \subseteq B$ be commutative rings and $C$ be a commutative $B$-algebra. Suppose $\sigma=\sum_{i} a_{i} \otimes b_{i} \otimes c_{i}$ is a C-two-cocycle with $m_{B, B}(\sigma)=1$ $\otimes_{B} 1 \otimes_{B}$ 1. Then under the map

$$
C \otimes C \otimes_{B} C \rightarrow(C \otimes C) \otimes_{C \otimes B}(C \otimes C)
$$

given by $x \otimes y \otimes_{B} z \rightarrow(x \otimes y) \otimes_{C \otimes B}(1 \otimes z)$ the element $\left\{1 \otimes m_{B}\right\}(\sigma)$ maps to a one-cocycle.

Proof. Applying $1 \otimes m_{B, B}$ to the associativity relation for $\sigma$ yields

$$
\sum_{i, j} a_{i} a_{j} \otimes b_{j} \otimes_{B} c_{j} b_{i} \otimes_{B} c_{i}=\sum_{i} a_{i} \otimes b_{i} \otimes_{B} 1 \otimes_{B} c_{i}
$$

since $m_{B, B}(\sigma)=1 \otimes_{B} 1 \otimes_{B} 1$ by assumption. Under the map

$$
C \otimes C \otimes_{B} C \otimes_{B} C \stackrel{\sim}{\rightarrow}(C \otimes C) \otimes_{C \otimes B}(C \otimes C) \otimes_{C \otimes B}(C \otimes C)
$$

given by

$$
w \otimes x \otimes_{B} y \otimes_{B} z \rightarrow(w \otimes x) \otimes_{C \otimes B}(1 \otimes y) \otimes_{C \otimes B}(1 \otimes z),
$$

(3.4a) transforms to

$$
\begin{aligned}
\sum_{i, j}\left(a_{j} \otimes b_{j}\right) \otimes_{C \otimes B}\left(a_{i} \otimes c_{j} b_{i}\right) \otimes_{C \otimes B}\left(1 \otimes c_{i}\right) \\
=\sum_{i}\left(a_{i} \otimes b_{i}\right) \otimes_{C \otimes B}(1 \otimes 1) \otimes_{C \otimes B}\left(1 \otimes c_{i}\right) .
\end{aligned}
$$

This establishes the lemma. 
THEOREM 3.5. Let $k \subseteq B$ be fields and $C$ be a commutative $B$-algebra. Suppose $\sigma$ is a $C$-two-cocycle with $m_{B, B}(\sigma)=1 \otimes_{B} 1 \otimes_{B} 1$. Then $\sigma$ is equivalent to a $B$-two-cocycle.

Proof. By Lemma 3.4, $\left\{1 \otimes m_{B}\right\}(\sigma)$ is a one-cocycle. Since $C \otimes C$ is a free $C \otimes B$-module, Theorem 3.1 and Lemma 3.2 imply

$$
\left\{1 \otimes m_{B}\right\}(\sigma)=\sum_{i, j} x_{i} \bar{x}_{j} \otimes y_{i} \otimes \bar{y}_{j}
$$

where $\delta=\Sigma_{i} x_{i} \otimes y_{i}$ is a unit in $C \otimes C$ with inverse $\bar{\delta}=\Sigma_{i} \bar{x}_{i} \otimes \bar{y}_{i}$ and $m_{C}(\delta)=1 . \sigma_{1} \sim \delta$ defines a $C$-two-cocycle $\sigma_{1}$ with $\left\{1 \otimes m_{B}\right\}\left(\sigma_{1}\right)=1 \otimes 1$ $\otimes_{B} 1$ since

$$
\left\{m_{B} \circ\left(m_{C} \otimes 1\right)\right\}(\sigma)=1 \otimes_{B} 1
$$

implies $m_{B}(\bar{\delta})=1 \otimes_{B} 1$. Since $B$ is a field, an application of $1 \otimes 1 \otimes m_{B}$ to the associativity relation for $\sigma_{1}$ demonstrates that $\sigma_{1}$ is in $C \otimes C \otimes B$.

By a similar argument using the analog of Lemma 3.4 for the map

$$
C \otimes_{B} C \otimes B \stackrel{\sim}{\rightarrow}(C \otimes B) \otimes_{B \otimes B}(C \otimes B)
$$

given by $x \otimes_{B} y \otimes z \rightarrow(x \otimes 1) \otimes_{B \otimes B}(y \otimes z)$, there exists a unit $\delta_{1}$ in $C \otimes B$ with $m_{C}\left(\delta_{1}\right)=1$ such that $\sigma_{2} \sim \delta_{1} \sigma_{1}$ defines a $C$-two-cocycle $\sigma_{2}$ with

$$
\left\{m_{B} \otimes 1\right\}\left(\sigma_{2}\right)=1 \otimes_{B} 1 \otimes 1 \text {. }
$$

Since $\delta_{1}$ is in $C \otimes B,\left\{1 \otimes m_{B}\right\}\left(\sigma_{2}\right)=1 \otimes 1 \otimes_{B} 1$.

By applying $m_{B} \otimes 1 \otimes 1$ to the associativity relation for $\sigma_{2}$ one may show that $\sigma_{2}$ is in $B \otimes C \otimes B$. That $\sigma_{2}$ is actually in $B \otimes B \otimes B$ then follows from an application of $1 \otimes m_{B} \otimes 1$ to the associativity relation for $\sigma_{2}$. Hence we are done.

We are now ready to prove:

TheORem 3.6. Let $k \subseteq B$ be fields and let $C$ be a commutative $B$-algebra. Suppose also that the natural map $m_{B}: C \otimes C \rightarrow C \otimes_{B} C$ induces a surjection on the groups of units. Then

$$
\{1\} \rightarrow \mathcal{H}^{2}(B) \stackrel{\iota^{*}}{\rightarrow} \mathcal{H} \mathcal{C}^{2}(C) \stackrel{r}{\rightarrow} \mathcal{H}^{2}(C / B)
$$

is exact.

Proof. The injectivity of $\iota^{*}$ follows from the corollary to Theorem 3.3. Hence to finish the proof we must show exactness at $\mathcal{H}^{2}(C)$.

Let $\sigma$ be a $C$-two-cocycle whose cohomology class is mapped to the trivial class under $r$, that is, $m_{B, B}(\sigma)$ is cohomologous to $1 \otimes_{B} 1 \otimes_{B} 1$ via an invertible element $\delta$ in $C \otimes_{B} C$. By hypothesis, we may lift $\delta$ to $\delta_{1}$ in $C \otimes C$. 
Then $\sigma_{1} \sim \delta^{\delta_{1}^{-1}} \sigma$ defines a $C$-two-cocycle $\sigma_{1}$ with $m_{B, B}\left(\sigma_{1}\right)=1 \otimes_{B} 1 \otimes_{B} 1$. Thus by Theorem 3.5 there is a $B$-two-cocycle $\sigma_{2}$ with $\sigma_{2}$ equivalent to $\sigma_{1}$, and hence equivalent to $\sigma$. This completes the proof.

Corollary 3.7. Let $k \subseteq B$ be fields and $C$ be a commutative $B$-algebra. Then

$$
\{1\} \rightarrow \mathcal{H}^{2}(B) \stackrel{\iota^{*}}{\rightarrow} \mathcal{H}^{2}(C) \stackrel{r}{\rightarrow} \mathcal{H}^{2}(C / B)
$$

is exact if either

(i) $B$ is a purely inseparable field extension of $k$, or

(ii) $B$ is a separable field extension of $k$ and $C$ is purely inseparable over $B$.

Proof. By the theorem, it suffices to show that either (i) or (ii) implies $m_{B}$ induces a surjection on units.

(i) Assume $B$ is purely inseparable over $k$. The kernel of $m_{B}$ is generated as a $C$-bimodule by $\{b \otimes 1-1 \otimes b \mid b$ is in $B\}$. When $B$ is purely inseparable over $k$, this is clearly an ideal generated by nilpotent elements and hence lies in the Jacobson radical of $C \otimes C$. The surjectivity on units thus follows since units may be lifted modulo the radical.

(ii) Since $C$ is a purely inseparable $B$-algebra (cf. [9]), the kernel of the multiplication map $C \otimes_{B} C \rightarrow C$ given by $x \otimes_{B} y \rightarrow x y$ is contained in the Jacobson radical of $C \otimes_{B} C$. Thus any unit of $C \otimes_{B} C$ may be written as $\alpha \otimes_{B} 1+\Sigma_{i} u_{i} \otimes_{B} v_{i}$ with $\alpha$ a unit in $C$ and $\Sigma_{i} u_{i} \otimes_{B} v_{i}$ nilpotent. If $\Sigma_{i} x_{i} \otimes y_{i}$ is a separability idempotent for $B$ over $k, \alpha \otimes 1+\sum_{i, j} u_{i} x_{j} \otimes y_{j} v_{i}$ is a unit of $C \otimes C$ which maps to $\alpha \otimes_{B} 1+\Sigma_{i} u_{i} \otimes_{B} v_{i}$. Therefore we are done.

REMARK. If we restrict our attention in Corollary 3.7 to the groups of units, we obtain well-known results on Amitsur cohomology. The reader is referred to Yuan [10, Theorems 3.4, 3.5].

Acknowledgement. The author is indebted to Moss Sweedler for several enlightening discussions during the preparation of this paper.

\section{REFERENCES}

1. S. A. Amitsur, Simple algebras and cohomology groups of arbitrary fields, Trans. Amer. Math. Soc. 97 (1959), 73-112.

2. A. J. Berkson, On Amitsur's complex and restricted Lie algebras, Trans. Amer. Math. Soc. 109 (1963), 430-443.

3. H. Cartan and S. Eilenberg, Homological algebra, Princeton Univ. Press, Princeton, N. J., 1956.

4. G. Hochschild, On the cohomology groups of an associative algebra, Ann. of Math. (2) 46 (1945), 58-67.

5. D. Riffelmacher, Multiplication alteration and related rigidity properties of algebras, Pacific J. Math. 71 (1977), 139-157.

6. A. Rosenberg and D. Zelinsky, Amitsur's complex for inseparable fields, Osaka Math. J. 14 (1962), 219-240. 
7. M. Sweedler, Groups of simple algebras, Inst. Hautes Études Sci. Publ. Math. 44 (1975), 79-189.

8. ___ Multiplication alteration by two-cocycles, Illinois J. Math. 15 (1971), 302-323.

9. __ Purely inseparable algebras, J. Algebra 35 (1975), 342-355.

10. S. Yuan, On the theory of p-algebras and the Amitsur cohomology groups for inseparable field extensions, J. Algebra 5 (1967), 280-304.

Departmient of Mathematics, Cornell Universtty, Ithaca, New Yorx 14853

Washington Opbrations, General Restarch Corporation, McLban, Viroinia 22101

Current address: Bell Laboratories, Holmdel, New Jersey 07733 